\title{
The photosensitizer verteporfin has light-independent anti- leukemic activity for Ph-positive acute lymphoblastic leukemia and synergistically works with dasatinib
}

\author{
Takanobu Morishita ${ }^{1}$, Fumihiko Hayakawa1, Keiki Sugimoto², Mizuho Iwase ${ }^{3}$, \\ Hideyuki Yamamoto${ }^{1}$, Daiki Hirano1, Yuki Kojima', Naoto Imoto', Tomoki Naoe ${ }^{4}$ \\ and Hitoshi Kiyoi ${ }^{1}$ \\ ${ }^{1}$ Department of Hematology and Oncology, Nagoya University Graduate School of Medicine, Nagoya, Japan \\ ${ }^{2}$ Fujii Memorial Research Institute, Otsuka Pharmaceutical Co., Ltd., Otsu, Japan \\ ${ }^{3}$ Department of Analytical Neurobiology, Faculty of Pharmacy, Meijo University, Nagoya, Japan \\ ${ }^{4}$ National Hospital Organization Nagoya Medical Center, Nagoya, Japan \\ Correspondence to: Fumihiko Hayakawa, email: bun-hy@med.nagoya-u.ac.jp \\ Keywords: drug screening, patient-derived xenograft, $\mathrm{Ph}^{+} \mathrm{ALL}$, verteporfin, drug repositioning \\ Received: July 10, $2016 \quad$ Accepted: July 23, $2016 \quad$ Published: August 02, 2016
}

\section{ABSTRACT}

Cell lines have been used for drug discovery as useful models of cancers; however, they do not recapitulate cancers faithfully, particularly from the viewpoints of microenvironmental independence. Patient-derived xenografts (PDX) are established by the transfer of primary tumor cells directly from patients into immunodeficient mice and can provide primary-like tumor cells of the amount needed at the desired time. We developed a high-throughput drug screening system using PDX cells and performed drug screening using the PDX cells of Philadelphia chromosome-positive acute lymphoblastic leukemia ( $\left.\mathrm{Ph}^{+} \mathrm{ALL}\right)$. We established four $\mathrm{Ph}^{+} \mathrm{ALL}$ PDX mice and performed high-throughput screening of 3440 compounds using leukemia cells from the PDX mice (PDX-cell screening). The profiles of drugs selected by PDX-cell screening were markedly different from those by screening using the $\mathrm{Ph}^{+} \mathrm{ALL}$ cell line. We found that verteporfin, an FDA-approved drug, exhibited strong PDX cell-specific cytotoxicity. In the validation assay, its GI $_{50}$ was $228 \mathrm{nM}, 395 \mathrm{nM}$, and $538 \mathrm{nM}$ in three PDX cells and $3.93 \mu \mathrm{M}, 2.11 \mu \mathrm{M}$, and 5.61 $\mu \mathrm{M}$ in three cell lines. Although verteporfin is a photosensitizer activated by photoirradiation, its cytotoxic effects were mediated by the light-independent production of reactive oxygen species; therefore, its antileukemic effects were also exerted in vivo without photoirradiation. Furthermore, it exhibited synergistic effects with dasatinib, an ABL kinase inhibitor. These results indicated the potential of verteporfin as a new anti-leukemic reagent.

\section{INTRODUCTION}

ABL kinase inhibitors are highly effective for BCR-ABL-positive leukemias such as chronic myeloid leukemia (CML) and Philadelphia chromosome-positive acute lymphoblastic leukemia $\left(\mathrm{Ph}^{+} \mathrm{ALL}\right)$. ABL kinase inhibitors keep CML in the chronic phase for a long period of time and markedly increase the complete remission rate of $\mathrm{Ph}^{+} \mathrm{ALL}$; however, the relapse of $\mathrm{Ph}^{+} \mathrm{ALL}$ is almost inevitable. [1] The only way to currently achieve a cure is to undergo allogeneic-stem cell transplantation (allo-SCT) during remission $[2,3]$; however, the therapy-related death rate of allo-SCT is high, and $\mathrm{Ph}^{+} \mathrm{ALL}$ is common among the elderly, who cannot receive allo-SCT. Therefore, there are still unmet medical needs for $\mathrm{Ph}^{+} \mathrm{ALL}$.

Target-based screening and phenotype-based screening represent the two major ways to develop drugs. In target-based screening, the target molecule of a certain disease, such as a particular kinase, is initially selected based on its knowledge of molecular pathology. Compounds are screened by evaluating their inhibitory effects on the enzymatic activity of the target molecule in vitro. Their effects on cell lines and mouse models are then investigated. This is a powerful method that is currently 
being used to develop many tyrosine kinase inhibitors including ABL kinase inhibitors; however, difficulties have been associated with identifying the true molecular targets of diseases in many cases, and inhibition of the target molecule does not always lead to anti-tumor effects. Even when the target molecule is successfully inhibited, antitumor effects may not be obtained due to the activation of an escape signal or evolution of resistant clones with drugresistant mutations. [4-6] Clonal evolution by the T315I mutation in BCR-ABL represents a severe obstacle for $\mathrm{Ph}^{+}$ ALL therapy. [7]

Phenotype-based screening using cell lines as cancer models have widely been used in the development of antitumor drugs. In this method, library compounds are added to the culture media of cell lines and selected based on their growth inhibitory effects on the cell lines. Most anticancer agents used in standard therapy for leukemia were developed by the 1970s. The development of innovative agents to replace previous standard therapies for leukemia using phenotype-based screening has not advanced for more than 30 years, suggesting the limitations of cell line-based screening and existing libraries for drug development. [8] Completely new libraries and novel cancer models to replace cell lines are now required for the serial development of innovative anti-cancer agents.

In order to overcome these limitations, we previously developed a new high-throughput drugscreening system using lymphoma cells obtained from patient-derived xenografts (PDX). PDX are established by the transfer of primary cancer cells directly from patients into immunodeficient mice. PDX faithfully maintain the characteristics of the parental tumors such as gene expression profiles, the mutational status including genome copy number variants, and metastatic potential even after several passages $[9,10]$. PDX also maintain hierarchy of the cancer cell differentiation status $[11,12]$ and drug responsiveness. [13] Furthermore, PDX may provide the required amount of primary-like cancer cells at the desired time. In the present study, we performed highthroughput screening of 3440 compounds using leukemia cells from $\mathrm{Ph}^{+} \mathrm{ALL}$ PDX (PDX-cell screening), and found that the photosensitizer, verteporfin, exhibited strong antileukemic effects both ex vivo and in vivo. These effects were mediated by oxidative stress and had synergism with dasatinib, an ABL kinase inhibitor clinically used for $\mathrm{Ph}^{+}$ ALL. These results indicated the potential of verteporfin as a new anti-leukemic reagent and PDX-cell screening as a novel strategy for the development of anti-cancer drugs.

\section{RESULTS}

\section{Establishment of ex vivo culturing of PDX cells}

Primary $\mathrm{Ph}^{+}$ALL cells obtained from the bone marrow of four patients were intravenously transplanted into NOD/SCID/ IL-2R $\gamma^{\text {null }}$ (NOG) mice. Patients' backgrounds and disease characteristics are summarized in Supplemental Table 1. All leukemia cells were successfully engrafted into mice. A total of $1.3 \times 10^{8}$ to $5.8 \times 10^{8}$ cells were obtained from one PDX mouse and the ratios of leukemia cells were 86.0 to $95.7 \%$ (Supplemental Table 2). PhLO cells were the most efficiently obtained cells.

PDX cells did not survive well without stromal cells ex vivo, and $\mathrm{S} 17$ cells, murine bone marrow stromal cells, were found to support the survival of $\mathrm{Ph}^{+} \mathrm{ALL}$ PDX cells in our previous study. [14] Therefore, we examined the survival of the PDX cells obtained in a co-culture with S17 cells. PhLO cells showed the highest survival rate (Figure 1A) and a slow growth rate with a doubling time of more than 14 days (Figure 1B).

\section{Verteporfin was specifically selected by PDX-cell screening}

We performed drug screening using PhLO cells co-cultured with S17 cells as described previously [15] (PDX-cell screening), and compared the profiles of the selected drugs to those of screening using ALL-1 cells, a $\mathrm{Ph}^{+}$ALL cell line (Cell-line screening). The library of 3440 compounds containing off-patent drugs was screened. Screening was performed using PhLO cells co-cultured with S17 cells, mono-cultured ALL-1 cells, and monocultured S17 cells, as shown in Figure 2A. All screenings were performed well with Z'-factors of $0.88,0.56$, and 0.93 and coefficient of variation values of $7.33 \%, 4.48 \%$, and $2.26 \%$, respectively.

All compounds were plotted on scattergrams, on which MTT values in ALL-1 cells (Figure 2B) or viabilities in PDX cells (Figure 2C) were set on the Y-axis and MTT values in S17 cells were set on the $\mathrm{X}$-axis. Compounds with MTT values in ALL-1 cells (Figure 2B) or viabilities in PDX cells less than 0.5 were discriminated as effective compounds in each screening. Effective compounds in one screening were plotted with red dots in another screening (Figure 2B and 2C). The profiles of the selected drugs were markedly different between PDX-cell screening and Cell-line screening. Cell-line screening was generally more sensitive than PDX-cell screening. The number of effective compounds in PDX-cell screening was 60 (Figure 2C), whereas 597 compounds were effective in Cell-line screening (Figure 2B). Only 37 compounds out of 597 effective compounds (6\%) in Cell-line screening were also effective in PDX- 
Table 1: Top 10 drugs selected by PDX screening.

\begin{tabular}{cccc} 
& PhLO viability (\%) & Relative MTT value of ALL1 & Relative MTT value of S17 \\
\cline { 2 - 4 } Pentoxifylline & 0 & 0.46 & 0.87 \\
Rilmenidine hemifumarate & 0 & 0.66 & 0.75 \\
AC-93253 iodide & 0 & 0.29 & 0.83 \\
Idarubicin & 0 & 0.26 & 0.82 \\
Chicago sky blue 6B & 0 & 0.91 & 0.55 \\
Pyrvinium pamoate & 0 & 0.34 & 0.38 \\
Aurantimycin A & 0 & 0.21 & 0.29 \\
Verteporfin & 0.01 & 1.08 & 0.3 \\
Stattic & 9.9 & 0.19 & 0.69
\end{tabular}

cell screening (Figure 2C), suggesting the difficulty associated with identifying effective compounds for PDX cells by Cell-line screening. On the other hand, 37 out of 60 effective compounds (62\%) in PDX-cell screening were also effective in Cell-line screening.

The top 10 compounds in PDX-cell screening were shown in Table 1. We selected verteporfin for further analysis. Verteporfin exhibited strong cytotoxicity in PDX-cell screening, but did not show significant growth inhibition in Cell-line screening (Figure 2B and 2C).
Verteporfin is an FDA-approved drug for the treatment of age-related macular degeneration (ARMD) and is suitable for being injected into humans.

Furthermore, we made a new library by selecting the top 200 compounds in PDX-cell screening using PhLO cells and subjected them to other PDX-cell screenings using PhLH and PhLK cells and Cell-line screening using NPhA1, ALL-1, and TCC-Y/sr cells. All cells were PDX cells or cell lines of $\mathrm{Ph}^{+}$ALL. PhLH and TCC-Y/ sr both had the T315I mutation of BCR-ABL, which
A

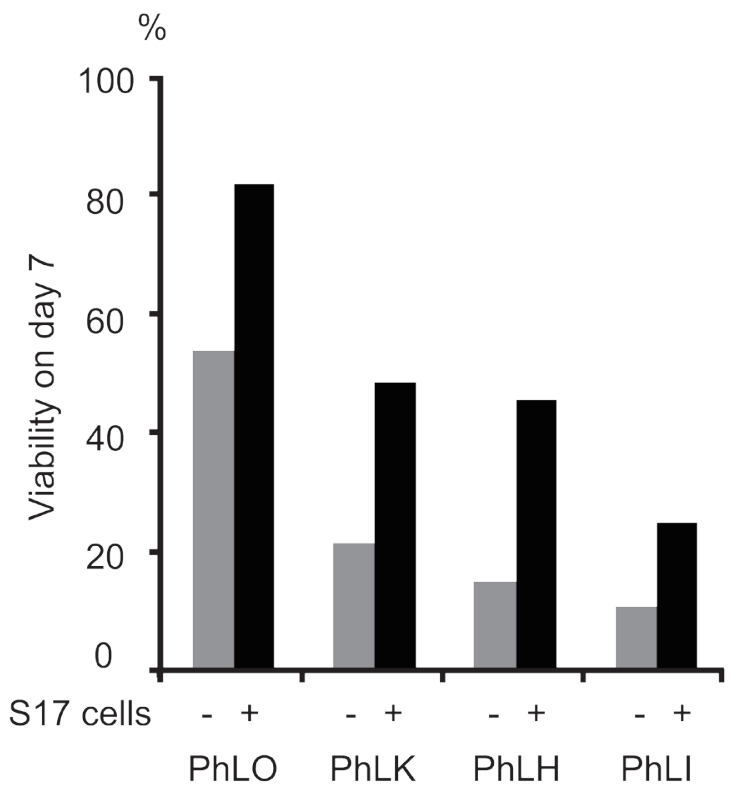

B

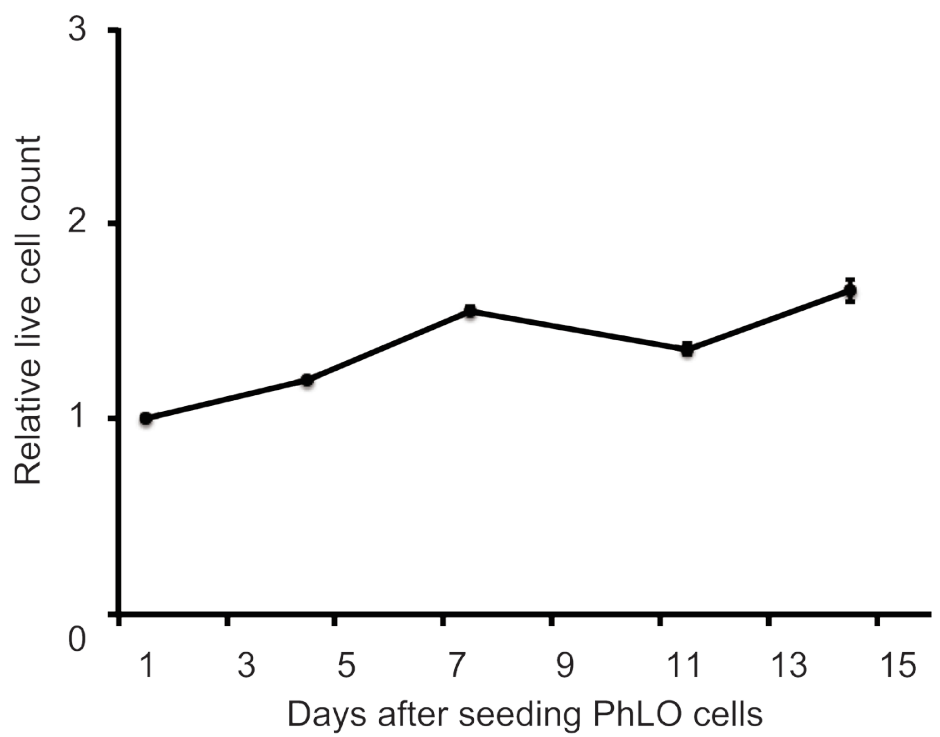

Figure 1: Establishment of an ex vivo culture of PDX cells. A. Survival improvements in PDX cells by a co-culture with stromal cells. PDX cells were cultured with or without S17 cells, as indicated. Viabilities were measured by DAPI staining and a flow cytometric analysis on day 7. B. The slow growth rate of $\mathrm{PhLO}$ cells ex vivo. $\mathrm{PhLO}$ cells were cultured with $\mathrm{S} 17$ cells as in A.. Live cell counts relative to that on day 1 were plotted on a line graph. Assays were performed in triplicate. Mean values were plotted with standard deviations. 
caused strong resistance to ABL kinase inhibitors. [7] The clinical and genetical backgrounds of the cell lines are summarized in Supplemental Table 3. We compared the drug sensitivity profiles of these cells. We evaluated drug effects in these Cell-line screenings with the same method as in PDX screenings, that is, with the image analyzer to make the assay conditions equal. The relationships between drug sensitivity profiles among PDX cells were weak, whereas those among cell lines were strong. The correlation coefficients between PhLO and PhLH, PhLO and $\mathrm{PhLK}$, and $\mathrm{PhLH}$ and PhLK were $0.215,0.375$, and 0.554 , respectively, while those between $\mathrm{NPhA} 1$ and ALL-1, NPhA1 and TCC-Y/sr, and ALL-1 and TCC-Y/sr were $0.817,0.909$, and 0.706 , respectively (Supplemental

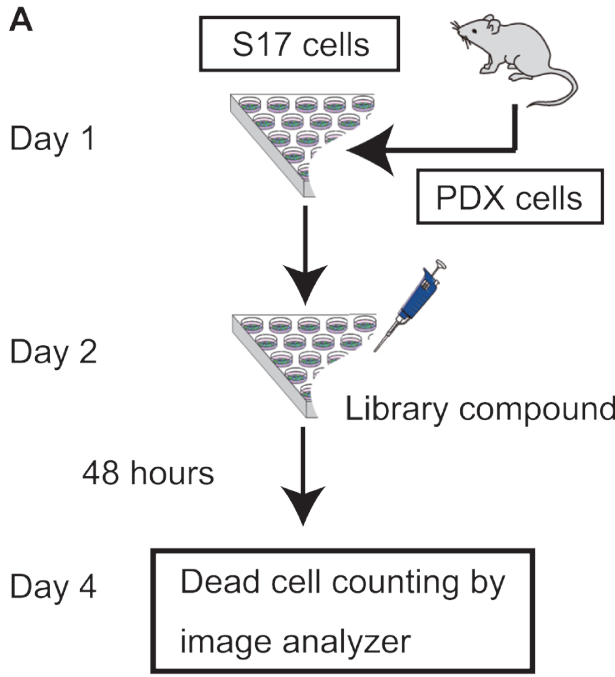

B

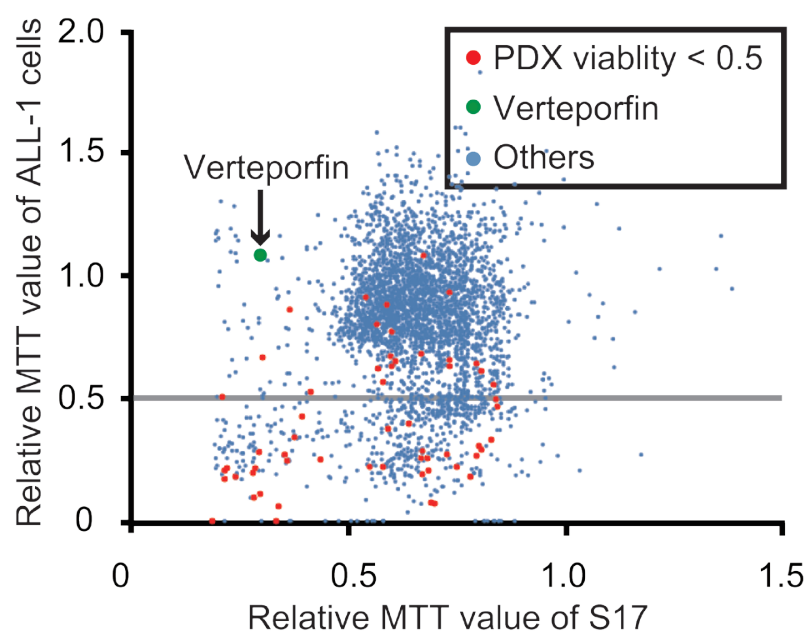

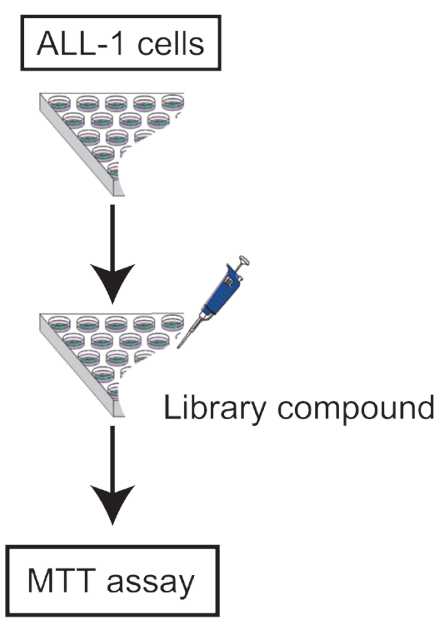

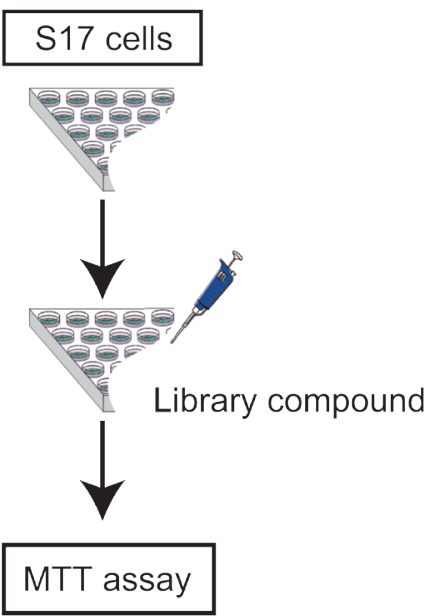

C

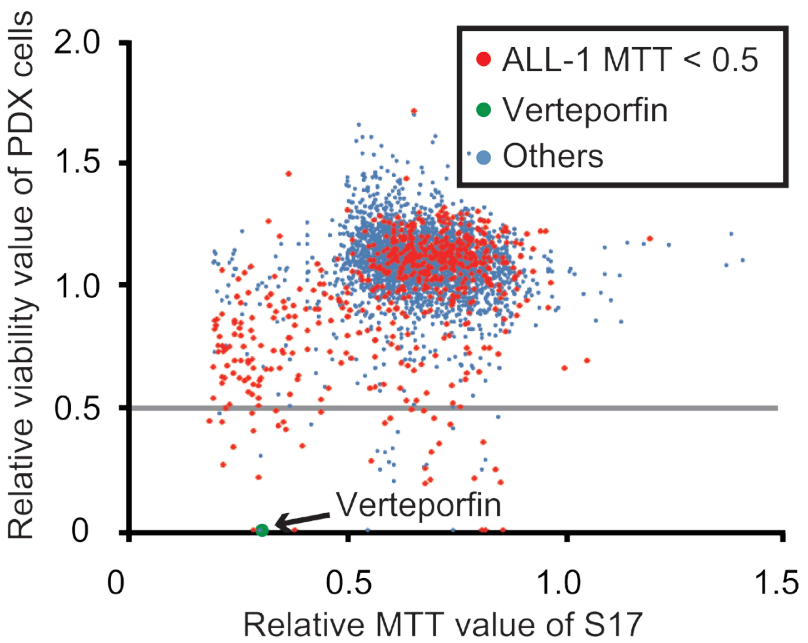

Figure 2: Comparison between PDX-cell screening and Cell-line screening. A. Flow chart of screenings. PhLO were sacrificed to obtain PDX cells on day 1. PDX cells $\left(1.0 \times 10^{4} /\right.$ well $)$ and S17 cells $\left(1.0 \times 10^{3} /\right.$ well $)$, ALL-1 cells $\left(1.0 \times 10^{4} /\right.$ well $)$ alone, and S17 cells $\left(1.0 \times 10^{3} /\right.$ well $)$ alone were seeded on 96 -well plates as indicated on day 1 . Drug-library compounds were added on day 2 . After 48 h, PDX cells were stained with Hoechst 33342 and PI. The viability of PDX cells was analyzed with an image analyzer. The growth of ALL-1 cells and S17 cells was measured by an MTT assay. B. Results of Cell-line screening. All compounds were plotted on a scattergram on which the relative MTT values of ALL-1 cells and S17 cells were set on the Y-axis and X-axis, respectively. The relative MTT values are relative values to the MTT value of the control cells treated with vehicle (DMSO). Effective compounds in PDX screening are plotted in red. Verteporfin is represented in green. The gray line indicates that the MTT value of ALL-1 is 0.5. Many of the red dots were plotted under the gray line, indicating that the effective compounds in PDX-cell screening were also frequently effective in Cell-line screening. C. Results of PDX-cell screening. All compounds were plotted on a scattergram on which the relative viabilities of PDX cells and relative MTT values of S17 cells were set on the Y-axis and X-axis, respectively. Effective compounds in Cell-line screening are plotted in red. Verteporfin is represented in green. The gray line indicates that the viability of PDX cells was 0.5 . Few of the red dots were plotted under the gray line indicating that effective compounds in Cell-line screening were rarely effective in PDX-cell screening. 
Figure 1). These results suggested that PDX cells had more diverse drug sensitivity profiles than those of cell lines.

\section{Verteporfin had strong anti-leukemic effects through light-independent ROS production}

The structural formula of verteporfin is shown in Figure 3A. Verteporfin is an example of a photosensitizer, which absorbs photons at specific wavelengths, induces the production of ROS, and then exerts cytotoxic effects. Verteporfin is currently used in photodynamic therapy for ARMD in which laser irradiation to the retina is performed 15 min after its intravenous administration in order to prevent angiogenesis. We compared the dose-dependency of its anti-leukemic effects among 3 PDX cells and 3 cell lines ex vivo. In addition to PhLO and ALL-1, PhLH, another $\mathrm{Ph}^{+}$ALL PDX cell, and TCC-Y/sr, another $\mathrm{Ph}^{+}$ ALL cell line, were examined. PhLH and TCC-Y/sr both have the T315I mutation in BCR-ABL. PDX cells were more sensitive to verteporfin than cell lines. The concentrations to cause $50 \%$ growth inhibition $\left(\mathrm{GI}_{50}\right)$ for $\mathrm{PhLO}, \mathrm{PhLH}$, and $\mathrm{PhLK}$ were $228 \mathrm{nM}, 395 \mathrm{nM}$, and $538 \mathrm{nM}$, respectively, whereas $\mathrm{GI}_{50}$ for ALL-1, TCC-Y/ sr, and NPhA1 were $3.93 \mu \mathrm{M}, 2.11 \mu \mathrm{M}$, and $5.61 \mu \mathrm{M}$, respectively (Figure 3B). These results were consistent with the results of the screenings. Although the T315I mutation in BCR-ABL leads to strong resistance to ABL kinase inhibitors [7], it did not cause significant resistance to verteporfin.

Since we performed all these experiments under minimum white fluorescent light, the cytotoxicity observed was considered to be independent of light. In order to clarify the mechanisms underlying light-independent cytotoxicity, we examined the type of cell death induced by verteporfin, and found that it induced apoptosis in all 4 PDX cells (Figure 3C). We speculated that verteporfin produced ROS to some extent without light activation, which lead to apoptosis in PDX cells because of their high sensitivity to oxidative stress. We found that verteporfin produced ROS in a light-independent manner in all 4 PDX cells to the same extent as menadione, a well-known ROS producer among various cells [16] (Figure 3D). In order to further confirm the involvement of oxidative stress in verteporfin-induced cytotoxicity, we investigated the effects of glutathione (GSH), a major reducing agent in cells, on its cytotoxicity. GSH significantly reduced the sensitivity of 2 out of 3 PDX cells to verteporfin (Figure $3 \mathrm{E}$ ), indicating the involvement of ROS production in the light-independent cytotoxicity of verteporfin.

\section{Verteporfin co-operatively worked with dasatinib ex vivo and in vivo}

ABL kinase inhibitors are the main components of combined chemotherapies for $\mathrm{Ph}^{+} \mathrm{ALL}$, and dasatinib has been proposed as the most effective for $\mathrm{Ph}^{+} \mathrm{ALL}$. [17] Therefore, we determined whether verteporfin had synergistic effects with dasatinib. A normalized isobologram and fraction affected-combination index plot were made in order to estimate drug interactions. Most combination data points fell on the lower left area of the isobologram (Figure 4A) and most combination index values were less than 1.0 (mean, 0.73 ; range, 0.28 1.34, Figure 4B and Supplemental Table 4). These results indicated the existence of synergistic anti-leukemic effects between verteporfin and dasatinib.

We next examined the anti-leukemic effect of verteporfin in vivo. In clinical use for ARMD, verteporfin is administered at a maximum of once a week. Therefore, in the first trial, we administered verteporfin $(10 \mathrm{mg} / \mathrm{kg})$ on alternate days from days 14 to 28 after the transplantation of leukemia cells; however, it did not lead to significant reductions in the number of leukemia cells (data not shown). Since its blood concentrations were very low, $3.7 \mathrm{nM}$ to $21.8 \mathrm{nM}, 24 \mathrm{~h}$ after its administration at 12.5 $\mathrm{mg} / \mathrm{kg}$ (Supplemental Figure 2A), continuous infusion appeared to be required in order for it to exert its antileukemia effects. We used osmotic pumps that delivered drugs by an osmotic process at a controlled rate for continuous infusion. The mean blood concentration of verteporfin after the implantation of osmotic pumps was $654 \mathrm{nM}$, approximately 3 folds higher than its $\mathrm{GI}_{50}$ in the ex vivo experiments (Supplemental Figure 2B). We assessed the in vivo effects of verteporfin using this system. Twelve NOG mice transplanted with $\mathrm{PhLO}$ cells were treated with vehicle, verteporfin, dasatinib, or a combination of both from days 22 to 28 , as shown in Figure 5A. The body weights of mice were similar among each group on day 28 , suggesting that drug toxicity was not severe in any group (Supplemental Figure 2C). Single therapies with verteporfin and dasatinib significantly reduced the leukemia cell ratio, and combined therapy further reduced the number of leukemia cells in the spleen (Figure 5B). Both of the single therapies had weaker anti-leukemic effects in bone marrow than in the spleen, however the combination therapy showed significantly enhanced effects (Figure 5C). These results indicated that verteporfin exhibited anti-leukemic activity in $\mathrm{Ph}^{+} \mathrm{ALL}$ when administered by itself and also in combination with dasatinib in vivo. We further investigated the effect of verteporfin on other 3 PDX models. Significant reduction of leukemia cells in spleen was observed in PhLI mice, whereas the effect was not significant in $\mathrm{PhLK}$ and $\mathrm{PhLH}$ mice (Figure 5D), suggesting sensitivity of verteporfin were various in vivo. We finally examined the effect of verteporfin on normal hematopoiesis and found that 

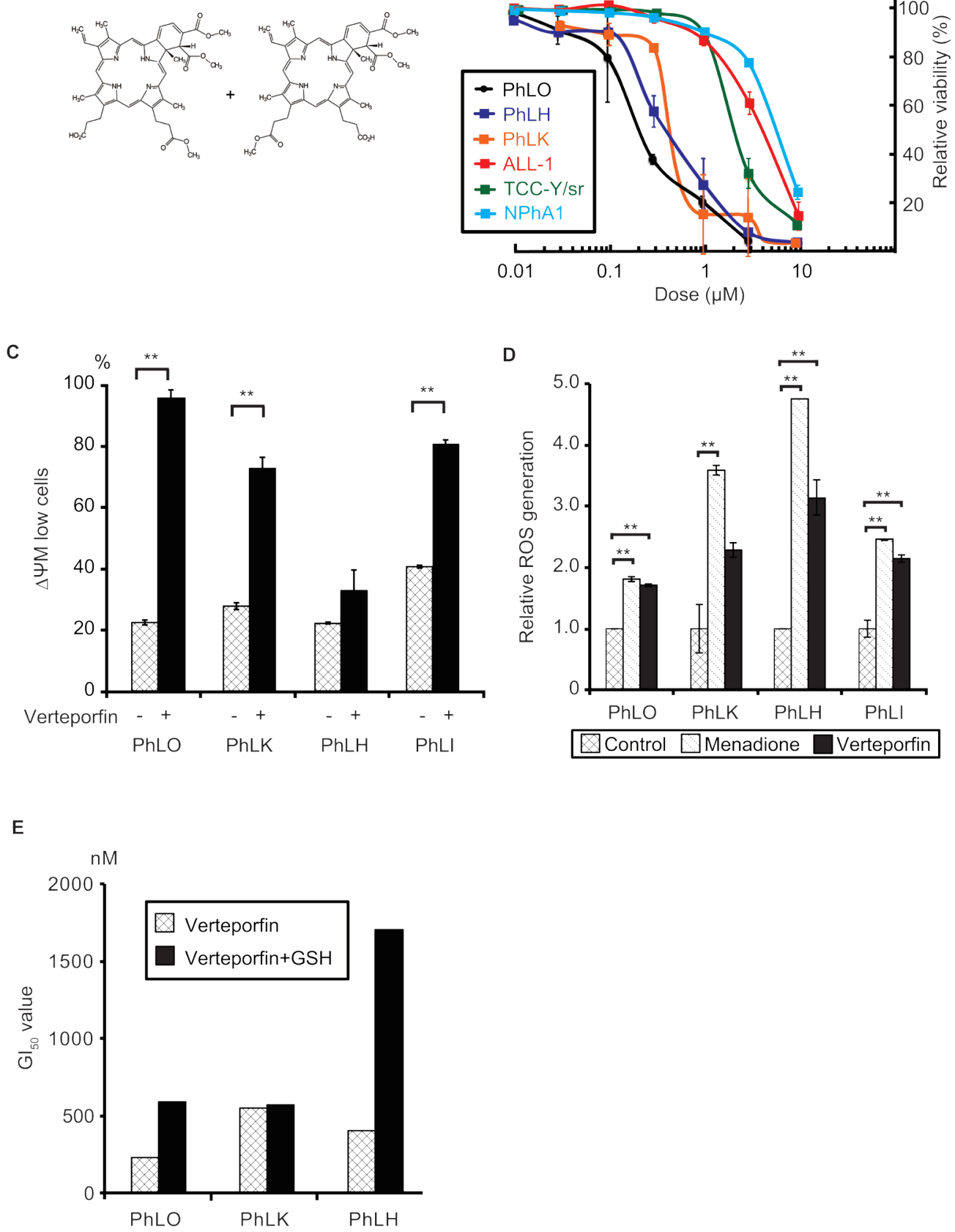

Figure 3: Verteporfin showed strong anti-leukemic effects through light-independent ROS production. A. Chemical structure of verteporfin. B. Dose-dependent anti-leukemic effects of verteporfin on PDX cells and $\mathrm{Ph}^{+}$ALL cell lines. PDX cells cocultured with S17 cells and mono-cultured cell lines were treated with the indicated concentrations of verteporfin for $48 \mathrm{~h}$. Viabilities were detected using a flow cytometer and presented as relative values to the viabilities of untreated cells. Each point represents the mean value for at least 3 independent experiments. Error bars show standard deviations. PDX cells were more sensitive to verteporfin than cell lines. C. Verteporfin reduced the mitochondrial membrane potential $(\triangle \Psi \mathrm{M})$ in PDX cells. The indicated PDX cells were treated with $1 \mu \mathrm{M}$ verteporfin, labeled with JC-1 reagents, and analyzed with a flow cytometer. The ratio of low $\triangle \Psi M$ PDX cells was plotted on a bar graph. D. Verteporfin induced ROS production in PDX cells. The indicated PDX cells were incubated with verteporfin $(2 \mu \mathrm{M})$ or menadione $(50$ $\mu \mathrm{M}$ ) for $3 \mathrm{~h}$. Menadione was used as the positive control of a ROS inducer. ROS production was measured by CellROX Green Oxidative Stress Reagents and plotted on a bar chart. E. GSH prevented the verteporfin-induced apoptosis in PDX cells. The indicated PDX cells co-cultured with S17 cells with or without $2 \mathrm{mM} \mathrm{GSH}$ in the culture medium were treated with the indicated dose of verteporfin as in B. $\mathrm{GI}_{50}$ of verteporfin of the indicated PDX cells with or without GSH were determined and plotted on a bar chart. **: $p<0.001$. GI ${ }_{50}$ were determined as results of at least 3 independent experiments. Error bars indicate standard deviations. GSH partly abolished the cytotoxicity of verteporfin in two of three PDX cells, suggesting that oxidative stress played a role in its cytotoxic effects. 
verteporfin did not significantly reduce the number of peripheral blood cells and bone marrow mononuclear cells (Supplemental Figure 3A and 3B), supporting the safety of verteporfin.

\section{DISCUSSION}

Using PDX-cell screening, we herein demonstrated that verteporfin exerted strong anti-tumor effects on $\mathrm{Ph}^{+}$ ALL. It produced ROS in leukemia cells and induced apoptosis, which was a different mechanism of action from conventional anti-tumor drugs. This drug had weak antileukemic effects on cell lines in spite of its strong antileukemic effects on PDX cells. Thus, Cell-line screening did not select this drug as a candidate anti-leukemic drug. This is an exceptional case. Cell-line screening was more sensitive than PDX-cell screening and discriminated many (597) compounds as effective; however, 94\% of these compounds were not effective for PDX cells (Figure 2C). This was markedly different from $62 \%$ of the effective compounds in PDX-cell screening also being effective in Cell-line screening (Figure 2B). Even when effective compounds were strictly selected, that is, only compounds that inhibited growth by more than $80 \%$ being selected as effective compounds in Cell-line screening, only 11 (12\%) out of 93 effective compounds were also effective in PDX-cell screening (data not shown). In addition,
23 compounds including verteporfin were effective in PDX cell screening, but were not in Cell-line screening. Although some of these differences in drug sensitivity may have been due to differences between the primary leukemia cells of ALL-1 cells and PhLO, based on the molecular pathological homogeneity of $\mathrm{Ph}^{+} \mathrm{ALL}$, many of these differences may be attributed to differences between PDX cells and cell lines. These results showed the difficulty in selecting the effective compounds for primary tumor cells by Cell-line screening and the superiority of PDX-cell screening in the development of anti-tumor drugs. Of note, the result that PDX cells had more diverse drug sensitivity profiles than those of cell lines (Supplemental Figure 1) was important. Since cell lines are tumor cells that are strongly selected based on growth rates under ex vivo culture, cell lines may lose the diversity of drug sensitivity during the establishment of cell lines from primary tumor cells. These results indicated the difficulty of choosing effective compounds for primary tumor cells by Cell-line screening and the superiority of PDX-cell screening in the development of anti-tumor drugs. The best method to search for compounds that commonly show strong anti-tumor effects in multiple PDX cells may be by multiple PDX-cell screenings.

We cannot currently conclude that PDX-cell screening is superior, because we need to determine whether this screening has the ability to select drugs
A

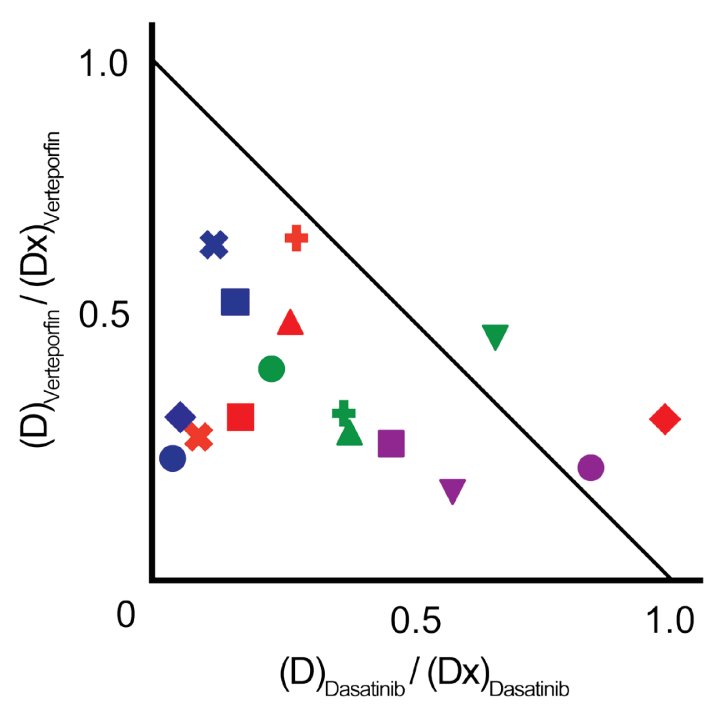

B

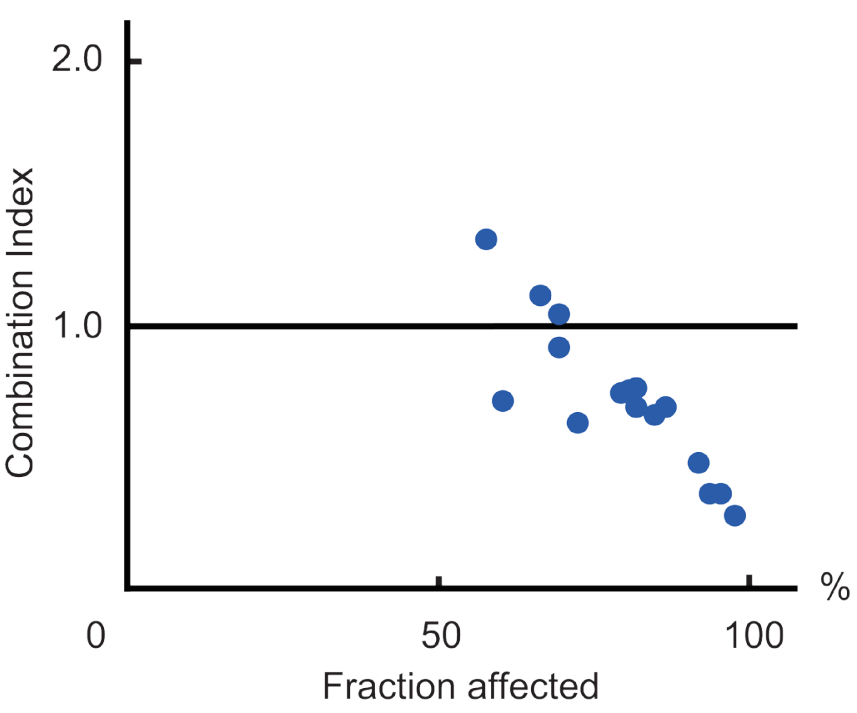

Figure 4: Verteporfin co-operated with dasatinib. A. A normalized isobologram between verteporfin and dasatinib. PhLO cells co-cultured with S17 cells were treated with 16 combinations of verteporfin (60nM, 120nM, 180nM, and 240nM) and dasatinib (12nM, $24 \mathrm{nM}, 36 \mathrm{nM}$, and $48 \mathrm{nM}$ ). The viabilities of cells treated with each combination were measured using a flow cytometer after $48 \mathrm{~h}$. Sixteen experimental data points are plotted. The list of markers of data points are shown in Supplemental Table 4. The normalized doses of dasatinib ((D) $\left.)_{\text {Dasatinib }} /(\mathrm{Dx})_{\text {Dasatinib }}\right)$ and verteporfin $\left((\mathrm{D})_{\text {Verteporfin }} /(\mathrm{Dx})_{\text {Verteporfin }}\right)$ are shown on the Y-axis and X-axis, respectively. The diagonal line represents $\mathrm{CI}=1.0$, the line of additive effects. The plot below the diagonal line represents synergistic effect, and above represents antagonistic effects. B. Fraction affected-CI plot. CI values and dead cell ratios in A. were plotted on the Y-axis and X-axis of a scatter gram, respectively. CI values were less than 1.0 in most of the plots (CI mean, 0.73; CI range, 0.28-1.34), indicating synergistic anti-leukemic effects between verteporfin and dasatinib. Experiments performed in duplicate and mean values were plotted. 
A

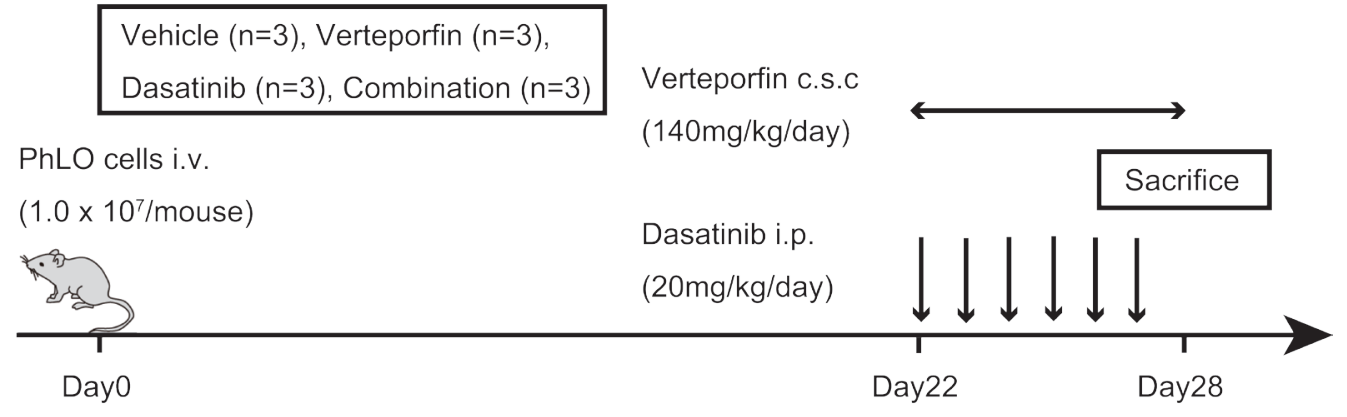

B

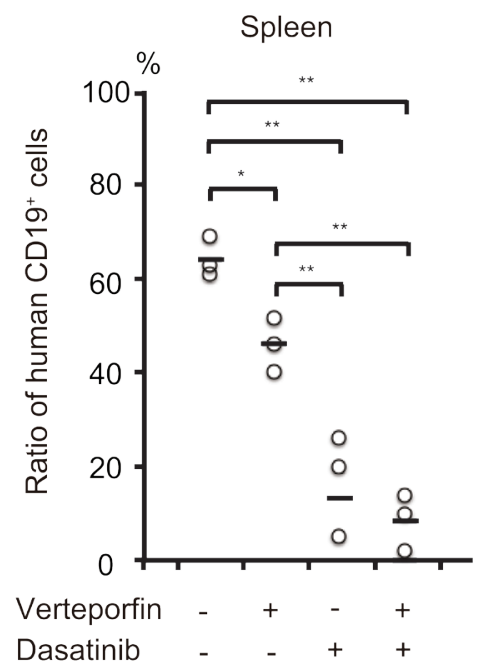

C

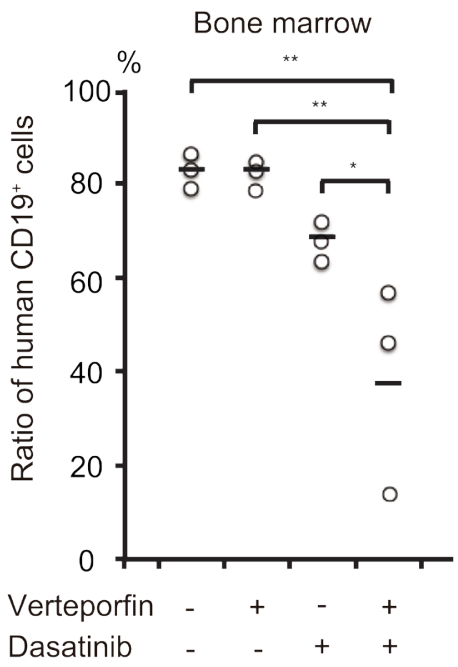

D

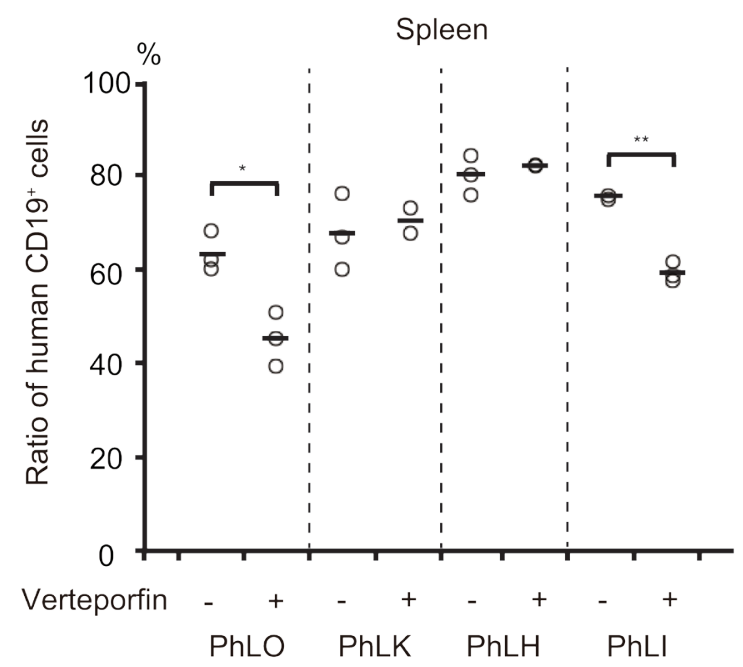

Figure 5: Verteporfin and dasatinib co-operated to reduce the number of leukemia cells in vivo. A. Schematic presentation of the treatment schedule. NOG mice transplanted with PhLO cells were treated with vehicle, verteporfin, dasatinib, or both from days 22 to 28 , as indicated. B. Leukemia cell number reductions in the spleen after the treatment with verteporfin and dasatinib. The leukemia cell ratio in spleen cells was analyzed using a flow cytometer with anti-human CD19 antibodies. Leukemia cell ratios in all mice were plotted. Single therapies with verteporfin and dasatinib significantly reduced the leukemia cell ratio. C. Leukemia cell number reductions in the bone marrow after the treatment with verteporfin and dasatinib. The leukemia cell ratio in bone marrow cells was analyzed as in B.. The combination therapy showed significantly enhanced effects, indicating synergistic effects between verteporfin and dasatinib in vivo. D. Comparison of in vivo effect of verteporfin among 4 PDX models. NOG mice were transplanted with the indicated PDX cells were treated with vehicle or verteporfin as in A.. Leukemia cell ratio in spleen was analyzed as in $\mathbf{B} . . * *: p<0.001, *: p<0.05$. The horizontal line is the mean of measurements. Abbreviations, i.v.: intravenous injection; c.s.c.: continuous subcutaneous injection; i.p.: intraperitoneal injection. 
more efficiently that have strong anti-tumor effects on actual cancer cells in patients. PDX-cell screening selects anti-cancer reagents that cannot be identified by Cellline screening, and provides new candidates for anticancer drug development. PDX-cell screening may detect anti-cancer drugs among existing drugs that have been overlooked for their anti-tumor activities.

We revealed that the light-independent antileukemic effects of verteporfin were attributed to ROS production without light. Pyruvinium pamoate, a ROS inducer, was also selected by PDX-cell screening using lymphoma PDX cells and exhibited strong anti-lymphoma activity in PDX mice in our previous study. Pyruvinium pamoate, similar to verteporfin, had strong anti-leukemic effects on PDX cells, but only had moderate effects on ALL-1 in this screening (Table 1). These results indicated that PDX cells were more sensitive to oxidative stress than cell lines. Reducing agents such as 2-mercaptoethanol are often added to culture media during the early stage of the establishment of cell lines including ALL-1. [18] The addition of reducing agents becomes unnecessary after the establishment of cell lines in many cases, suggesting that cell lines obtain resistance to oxidative stress during their establishment. PDX-cell screening may be a useful tool for discovering oxidative-stress-inducing drugs that are hard to select using Cell-line screening.

Verteporfin is activated by light, most efficiently at a wavelength of $690 \mathrm{~nm}$, and produces ROS in cells. Its cytotoxicity for some cancer cells such as pancreatic cancer, breast cancer, and leukemia has been reported; however, its cytotoxicity had been activated by light in all these cases. [19-21] Therefore, pioneering cancer therapy using verteporfin has been localized therapy combined with photoirradiation. [19] This is the first study on the light-independent cytotoxicity of verteporfin. This effect has been overlooked for a long period of time despite intensive investigations being conducted on this drug, possibly because it had weak cytotoxicity for cell lines and its cytotoxicity for primary tumor cells was hard to estimate. This result will promote the application of this drug to systemic chemotherapy for cancer and may markedly expand its potential uses.

Verteporfin was originally designed to be excreted rapidly after photoirradiation in order to prevent side effects such as photodermatosis. Therefore, the half-life of this drug is short at $5.6 \mathrm{~h}$. Verteporfin is an FDA-approved drug for ARDM; however, its approved usage is for single intravenous administration. Repetitive administration is only permitted one time with an interval at least one week. Since verteporfin did not exert its anti-leukemic effects in vivo with an alternate day administration protocol (data not shown), non-approved usage such as continuous infusion is needed when verteporfin is applied to the treatment of leukemia. No safety information for such usage is currently available, and therefore, a phase I study is needed.
The anti-leukemic effects of this drug in vivo were not as strong as those of dasatinib, and this drug was not effective in 2 out of 4 PDX models, PhLK and PhLH (Figure 5D). These will be because the blood concentration of verteporfin was not very high, only approximately 3-fold higher than its $\mathrm{GI}_{50}$ for PhLO cells. $\mathrm{GI}_{50}$ for PhLK and PhLH were about 2.4 and 1.7 times higher than PhLO (Figure 3B), which would lead to verteporfin-resistance of these mice model. Due to the poor solubility of verteporfin and the limited volume of the osmotic pump $(200 \mu \mathrm{l})$, we were unable to administer more in this system. The development of a better method for dissolving verteporfin will enable higher blood concentrations and stronger antileukemic effects in vivo.

Verteporfin had synergistic anti-leukemic effects with dasatinib. Previous studies reported the involvement of ABL kinase in resistance to oxidative stress. The nuclear translocation of c-ABL and activation of early growth response protein 1 by c-ABL have been shown to contribute resistance to oxidative stress. [22, 23] A previous study demonstrated that adaphostin-induced oxidative stress overcame resistance to imatinib, another clinically used ABL kinase inhibitor, in $\mathrm{Ph}^{+} \mathrm{ALL}$. [24] The inhibition of ABL kinase by dasatinib may attenuate the tolerance of leukemia cells to oxidative stress, which may function co-operatively with verteporfin-induced oxidative stress. The uptake of verteporfin by the primary cells of CML, another leukemia developed by BCR-ABL, is greater than that by the normal mononuclear cells of peripheral blood and bone marrow, which further explains their co-operation in vivo.

In summary, we herein performed PDX-cell screening using the PDX cells of $\mathrm{Ph}^{+}$ALL, identified oxidative stress as an important anti-leukemic mechanism that co-operatively functions with ABL kinase inhibition, and discovered verteporfin as an anti-leukemic ROS inducer. By introducing the phenotype of primary cancer cells at the earlier in vitro stages of drug development, PDX-cell screening sheds new light on anti-cancer drug development.

\section{MATERIALS AND METHODS}

\section{Cells}

S17 cells, a murine bone marrow stromal cell lines, were previously described. [14] S17 cells were cultured in Roswell Park Memorial Institute medium (RPMI) supplemented with $10 \%$ fetal bovine serum (FBS) for maintenance. ALL-1 cells, TCC-Y/sr cells, and NPhA1 were maintained in 10\% FBS-containing Iscove's modified Dulbecco's medium (ISCOVE) and 10\% FBS-containing RPMI medium, respectively. [25, 26] All cell lines have not been tested for authentication in our laboratory. 


\section{Reagents and antibodies}

Verteporfin and reduced-form GSH were purchased from Wako Pure Chemical Industries (Osaka, Japan). Dasatinib and menadione was obtained from Chemscene (Princeton, NJ, USA) and Sigma Aldrich (St. Louis, MO, USA), respectively. An anti-human CD19 antibody and anti-mouse CD45 antibody were purchased from BD Biosciences (San Jose, CA, USA).

\section{Compound library}

A library of 3440 compounds mainly consisting of off-patent drugs and pharmacologically active reagents was kindly provided by The Drug Discovery Initiative (The University of Tokyo, Tokyo, Japan) and described previously. [15]

\section{Establishment of PDX cells}

$\mathrm{PhLO}$ and $\mathrm{PhLK}$ cells were established in a previous study. [12] PhLH and PhLI were established as described previously. $[12,14,15]$ Briefly, primary $\mathrm{Ph}^{+} \mathrm{ALL}$ cells $\left(6.8-20 \times 10^{6}\right.$ cells $)$ were injected into the tail vein of non-irradiated 8-week-old male NOG mice. To prevent human $\mathrm{T}$ cell expansion, $0.1 \mathrm{mg}$ anti-CD3 antibody (Janssen Pharmaceutical, Tokyo, Japan) was injected on the same day. For the in vivo passage of PDX cells, BM cells or spleen cells ( 5 X $10^{6}$ cells) from a PDX mouse were injected into another NOG mouse with an 8-week interval. Primary cells were collected from patients after obtaining written informed consent, and the Institutional Review Board of Nagoya University Graduate School of Medicine approved this study.

\section{PDX-cell screening}

S17 cells $\left(1.0 \times 10^{3} /\right.$ well $)$ and PhLO cells $(1.0 \times$ $10^{4} /$ well) were seeded on 96-well plates in 10\% FBScontaining RPMI medium on day 1 . On day 2 , the library compounds ( $2 \mu \mathrm{M}$ each) were added to each well. After 48 hours (h) on day 4, total and dead PDX cells were stained with Hoechst 33342 and Propidium Iodide (PI), respectively. Total and dead PDX cells were counted separately from S17 cells using an Array Scan VTI HCS image analyzer (Thermo Fisher Scientific, Waltham, MA, USA) as described previously. [15] Since the library contained some photosensitive compounds, all experiments were performed under conditions that avoided sunlight and laser light.

\section{Cell-line screening}

ALL-1 cells $\left(1.0 \times 10^{4} /\right.$ well $)$ and S17 cells $(1.0 \times$ $10^{3} /$ well) were seeded on 96-well plates on day 1 . Library compounds were added to each well on day 2. MTT assays were performed using Cell Counting Kit-8 reagent (Dojindo Laboratories, Kumamoto, Japan) on day 4.

\section{Detection of apoptosis and ROS generation}

These assays were performed as described previously. [15]

\section{Estimation of drug interactions}

PDX cells co-cultured with S17 cells were treated with 16 combinations of verteporfin $(60 \mathrm{nM}, 120 \mathrm{nM}$, $180 \mathrm{nM}$, and $240 \mathrm{nM})$ and dasatinib (12 nM, $24 \mathrm{nM}, 36$ $\mathrm{nM}$, and $48 \mathrm{nM}$ ). The viabilities of cells treated with each combination were measured after $48 \mathrm{~h}$ using FACS Aria flow cytometer (BD Biosciences, San Jose, CA, USA). In order to estimate drug interaction between verteporfin and dasatinib, a normalized isobologram and fraction affectedcombination index (CI) plot were made using CompuSyn software (ComboSyn, Paramus, NJ, USA). [27] CI values greater than 1.0 indicated antagonistic effects, equal to 1.0 additive effects, and below 1.0 synergistic effects.

\section{Estimation of in vivo drug effects}

$\mathrm{PhLO}$ cells $\left(1.0 \times 10^{7}\right.$ /mouse $)$ were injected intravenously into 6-week-old male NOG mice, which were then treated with vehicle, verteporfin (140 milligram $(\mathrm{mg}) / \mathrm{kilogram}(\mathrm{kg}) /$ day), dasatinib $(20 \mathrm{mg} / \mathrm{kg} /$ day $)$, and a combination of these drugs from days 22 to 28 . Verteporfin was administered by continuous subcutaneous infusion (c.s.c.) using Alzet osmotic pumps (Alzet, Cupertino, CA, USA). An intraperitoneal injection (i.p.) was performed for dasatinib. All mice were sacrificed on day 28 and the chimerism of leukemia cells was investigated by flow cytometer using an anti-human CD19 antibody and antimouse CD45 antibody. Blood concentrations of verteporfin were calculated by LCMS-2020 (Shimadzu Corporation, Kyoto, Japan). All animal experiments were approved by the Nagoya University Animal Ethics Committee.

\section{Statistical analyses}

Differences between two groups were analyzed with the Student's $t$-test. Differences between more than three groups were examined with the Tukey-Kramer test. Statistical analyses were performed by R software (R Foundation for Statistical Computing, Vienna, Austria) 
and $p<0.05$ was considered significant.

\section{ACKNOWLEDGMENTS}

We thank the Drug Discovery Initiative (The Tokyo University, Tokyo, Japan) for providing the Prestwick and Lopack Chemical Library. We are very grateful to Yoko Matsuyama and Chika Wakamatsu for their technical assistance.

\section{CONFLICTS OF INTEREST}

K.S. is an employee of Otsuka Pharmaceutical Co. Ltd., T.N. received research funding from Fujifilm Corporation. H.K. received research funding from Chugai Pharmaceutical Co. LTD., Bristol-Myers Squibb, KyowaHakko Kirin Co. LTD., Zenyaku Kogyo Company LTD., and Fujifilm Corporation. The other authors have no potential conflicts of interest.

\section{GRANT SUPPORT}

This work was supported by MHLW KAKENHI Grant Number H22-3jigan-Ippan-010 and H26Kakushintekigan-Ippan-133, and JSPS KAKENHI Grant Numbers 23591381, 25118711, 25293218, 25670449, and 15K09472. This work was also supported by the Program to Disseminate Tenure Tracking System, MEXT, Japan and the Platform for Drug Discovery, Informatics, and Structural Life Science from the Ministry of Education, Culture, Sports, Science and Technology, Japan.

\section{Editorial note}

This paper has been accepted based in part on peerreview conducted by another journal and the authors' response and revisions as well as expedited peer-review in Oncotarget.

\section{REFERENCES}

1. Bauer RC, Sanger J, Peschel C, Duyster J and von Bubnoff $\mathrm{N}$. Sequential inhibitor therapy in CML: in vitro simulation elucidates the pattern of resistance mutations after secondand third-line treatment. Clinical cancer research. 2013; 19:2962-2972.

2. Fielding AK, Rowe JM, Richards SM, Buck G, Moorman AV, Durrant IJ, Marks DI, McMillan AK, Litzow MR, Lazarus HM, Foroni L, Dewald G, Franklin IM, Luger SM, Paietta E, Wiernik PH, et al. Prospective outcome data on 267 unselected adult patients with Philadelphia chromosome-positive acute lymphoblastic leukemia confirms superiority of allogeneic transplantation over chemotherapy in the pre-imatinib era: results from the
International ALL Trial MRC UKALLXII/ECOG2993. Blood. 2009; 113:4489-4496.

3. Wang L, Wang Y, Tang W, Dou H-B, Shan J-H and Hu $\mathrm{J}$. The superiority of allogeneic hematopoietic stem cell transplantation from unrelated donor over chemotherapy for adult patients with high-risk acute lymphoblastic leukemia in first remission. International Journal of Hematology. 2013; 98:569-577.

4. Koppikar P, Bhagwat N, Kilpivaara O, Manshouri T, Adli M, Hricik T, Liu F, Saunders LM, Mullally A, AbdelWahab O, Leung L, Weinstein A, Marubayashi S, Goel A, Gonen M, Estrov Z, et al. Heterodimeric JAK-STAT activation as a mechanism of persistence to JAK2 inhibitor therapy. Nature. 2012; 489:155-159.

5. Donato NJ, Wu JY, Stapley J, Gallick G, Lin H, Arlinghaus $\mathrm{R}$ and Talpaz M. BCR-ABL independence and LYN kinase overexpression in chronic myelogenous leukemia cells selected for resistance to STI571. Blood. 2003; 101:690698.

6. Bewry NN, Nair RR, Emmons MF, Boulware D, Pinilla-Ibarz J and Hazlehurst LA. Stat3 contributes to resistance toward BCR-ABL inhibitors in a bone marrow microenvironment model of drug resistance. Molecular cancer therapeutics. 2008; 7:3169-3175.

7. von Bubnoff N, Schneller F, Peschel C and Duyster J. $\mathrm{BCR}-\mathrm{ABL}$ gene mutations in relation to clinical resistance of Philadelphia-chromosome-positive leukaemia to STI571: a prospective study. The Lancet. 2002; 359:487-491.

8. Wilding JL and Bodmer WF. Cancer cell lines for drug discovery and development. Cancer research. 2014; $74: 2377-2384$.

9. Zhao X, Liu Z, Yu L, Zhang Y, Baxter P, Voicu H, Gurusiddappa S, Luan J, Su JM, Leung HC and Li XN. Global gene expression profiling confirms the molecular fidelity of primary tumor-based orthotopic xenograft mouse models of medulloblastoma. Neuro-oncology. 2012; 14:574-583.

10. DeRose YS, Wang G, Lin YC, Bernard PS, Buys SS, Ebbert MT, Factor R, Matsen C, Milash BA, Nelson E, Neumayer L, Randall RL, Stijleman IJ, Welm BE and Welm AL. Tumor grafts derived from women with breast cancer authentically reflect tumor pathology, growth, metastasis and disease outcomes. Nature medicine. 2011; 17:15141520.

11. Bonnet D and Dick JE. Human acute myeloid leukemia is organized as a hierarchy that originates from a primitive hematopoietic cell. Nature medicine. 1997; 3:730-737.

12. Tanizaki R, Nomura Y, Miyata Y, Minami Y, Abe A, Hanamura A, Sawa M, Murata M, Kiyoi H, Matsushita $\mathrm{T}$ and Naoe T. Irrespective of CD34 expression, lineagecommitted cell fraction reconstitutes and re-establishes transformed Philadelphia chromosome-positive leukemia in NOD/SCID/IL-2Rgammac-/- mice. Cancer science. 2010; 101:631-638. 
13. Hidalgo M, Bruckheimer E, Rajeshkumar NV, GarridoLaguna I, De Oliveira E, Rubio-Viqueira B, Strawn S, Wick MJ, Martell J and Sidransky D. A pilot clinical study of treatment guided by personalized tumorgrafts in patients with advanced cancer. Molecular cancer therapeutics. 2011; 10:1311-1316.

14. Kuwatsuka Y, Minami M, Minami Y, Sugimoto K, Hayakawa F, Miyata Y, Abe A, Goff DJ, Kiyoi H and Naoe T. The mTOR inhibitor, everolimus (RAD001), overcomes resistance to imatinib in quiescent $\mathrm{Ph}$-positive acute lymphoblastic leukemia cells. Blood cancer journal. 2011; 1:e17.

15. Sugimoto K, Hayakawa F, Shimada S, Morishita T, Shimada K, Katakai T, Tomita A, Kiyoi H and Naoe T. Discovery of a drug targeting microenvironmental support for lymphoma cells by screening using patient-derived xenograft cells. Scientific reports. 2015; 5:13054.

16. Beck R, Pedrosa RC, Dejeans N, Glorieux C, Leveque P, Gallez B, Taper H, Eeckhoudt S, Knoops L, Calderon $\mathrm{PB}$ and Verrax J. Ascorbate/menadione-induced oxidative stress kills cancer cells that express normal or mutated forms of the oncogenic protein Bcr-Abl. An in vitro and in vivo mechanistic study. Invest New Drugs. 2011; 29:891900 .

17. Talpaz M, Shah NP, Kantarjian H, Donato N, Nicoll J, Paquette R, Cortes J, O'Brien S, Nicaise C, Bleickardt E, Blackwood-Chirchir MA, Iyer V, Chen TT, Huang F, Decillis AP and Sawyers CL. Dasatinib in imatinib-resistant Philadelphia chromosome-positive leukemias. N Engl J Med. 2006; 354:2531-2541.

18. Epstein AL and Kaplan HS. Feeder layer and nutritional requirements for the establishment and cloning of human malignant lymphoma cell lines. Cancer research. 1979; 39:1748-1759.

19. Huggett MT, Jermyn M, Gillams A, Illing R, Mosse S, Novelli M, Kent E, Bown SG, Hasan T, Pogue BW and Pereira SP. Phase I/II study of verteporfin photodynamic therapy in locally advanced pancreatic cancer. British journal of cancer. 2014; 110:1698-1704.
20. Lo VC, Akens MK, Moore S, Yee AJ, Wilson BC and Whyne CM. Beyond radiation therapy: photodynamic therapy maintains structural integrity of irradiated healthy and metastatically involved vertebrae in a pre-clinical in vivo model. Breast cancer research and treatment. 2012; 135:391-401.

21. Jamieson $\mathrm{CH}, \mathrm{McD}$ onald $\mathrm{WN}$ and Levy JG. Preferential uptake of benzoporphyrin derivative by leukemic versus normal cells. Leukemia research. 1990; 14:209-219.

22. Nihira K, Taira N, Miki Y and Yoshida K. TTK/Mps1 controls nuclear targeting of c-Abl by 14-3-3-coupled phosphorylation in response to oxidative stress. Oncogene. 2008; 27:7285-7295.

23. Stuart JR, Kawai H, Tsai KK, Chuang EY and Yuan ZM. c-Abl regulates early growth response protein (EGR1) in response to oxidative stress. Oncogene. 2005; 24:80858092.

24. Chandra J, Tracy J, Loegering D, Flatten K, Verstovsek S, Beran M, Gorre M, Estrov Z, Donato N, Talpaz M, Sawyers C, Bhalla K, Karp J, Sausville E and Kaufmann SH. Adaphostin-induced oxidative stress overcomes BCR/ABL mutation-dependent and -independent imatinib resistance. Blood. 2006; 107:2501-2506.

25. Cesano A, O'Connor R, Lange B, Finan J, Rovera G and Santoli D. Homing and progression patterns of childhood acute lymphoblastic leukemias in severe combined immunodeficiency mice. Blood. 1991; 77:2463-2474.

26. Kano Y, Akutsu M, Tsunoda S, Mano H, Sato Y, Honma $\mathrm{Y}$ and Furukawa Y. In vitro cytotoxic effects of a tyrosine kinase inhibitor STI571 in combination with commonly used antileukemic agents. Blood. 2001; 97:1999-2007.

27. Chou TC. Theoretical basis, experimental design, and computerized simulation of synergism and antagonism in drug combination studies. Pharmacol Rev. 2006; 58:621681. 